\title{
DETERMINACIÓN DE LAS DIFERENCIAS EN LOS RESULTADOS DE DOS PROCEDIMIENTOS DE EJECUCIÓN DEL ENSAYO PROCTOR ESTÁNDAR
}

DETERMINATION OF THE DIFFERENCES IN RESULTS OF TWO REALIZATION PROCEDURES OF THE STANDARD PROCTOR TEST

\author{
Joseín Hernández Córdova \\ ing.josein.hdez@gmail.com \\ René Sebastián Mora Ortiz \\ rene.mora@ujat.mx
}

Emmanuel Munguía Balvanera balvanerae@hotmail.com 
Para citar este artículo:

Hernández Córdova, J., Mora Ortiz, R. S., \& Munguía Balvanera, E. (2020). Determinación de las diferencias en los resultados de dos procedimientos de ejecución del ensayo proctor estándar. ESPACIO I+D, INNOVACIÓN MÁS DESARROLLO, 9(24). https://doi. org/10.31644/IMASD.24.2020.a08

\section{RESUMEN}

El grado de compactación de los suelos se evalúa a través de dos parámetros: su peso específico seco máximo $\left(\gamma_{\text {dmax }}\right)$ y su humedad óptima $\left(\mathrm{w}_{\mathrm{opt}}\right)$, los cuales se pueden determinar en el laboratorio a través de diversos métodos. Una de las técnicas de mayor uso a nivel mundial es el ensayo Proctor estándar. El procedimiento clásico de este ensayo establece la construcción de una curva de compactación utilizando una sola porción de suelo que se compacta varias veces durante todo el procedimiento. Sin embargo, en la ejecución habitual de esta prueba ha surgido la variante de utilizar varias porciones de suelo en lugar de una sola. El objetivo de esta investigación es aportar información acerca de las diferencias en los resultados obtenidos entre estas dos variantes de la prueba Proctor estándar. Para lograrlo, en un primer grupo de ensayos se determinaron los parámetros óptimos de compactación de dos suelos diferentes del estado de Tabasco siguiendo el procedimiento clásico del ensayo Proctor Estándar. Estos resultados sirvieron de control. A continuación, en un segundo grupo de ensayos y para los mismos suelos, se repitieron las pruebas Proctor estándar pero utilizando varias porciones de suelo en lugar de una sola, esto con la finalidad de que cada porción solo se compactara una vez. Todos los ensayos Proctor se realizaron por triplicado y los resultados se ajustaron mediante una línea polinómica. Comparada con los resultados del ensayo Proctor estándar clásico, la variante que utiliza varias porciones de suelo generó un descenso de alrededor del 1\% en los valores de los pesos específicos secos máximos, y aumentos en las humedades óptimas en torno al 1.3\%. Estas diferencias se originan debido al proceso de preparación de las muestras de suelo en la variante del ensayo Proctor.

\section{Palabras clave}

Proctor Estándar; peso específico seco; contenido de agua; recompactación. 
Compaction is a soil densification process in which its resistance and load capacity are increased. The degree compaction of soils is evaluated through two parameters: its maximum dry specific weight $\left(\gamma_{\text {dmáx }}\right)$ and its optimal humidity $\left(\mathrm{w}_{\mathrm{opt}}\right)$, these parameters can be determined thought many laboratory techniques. Globally, one of the most used is the Standard Proctor test. The standard process of this test established the definition of one compaction curve using values from a single soil sample after being compacted several times during the procedure. However, in the usual implementation of this test has emerged a variation of the process, to define the compaction curve using more than one sample soil. The goal of this investigation is to provide information about the discordance of the resulting values from the two different techniques of the Standard Proctor test. To accomplished this, over a fist group of samples, it was determined the optimal compaction parameter of two different soil types from the Mexican state of Tabasco after following the standardized process of the Standard Proctor test. These first results served as control values. Subsequently, the second group of samples of the same soil types from the first procedure was tested again with the Standard Proctor test, but now, over several soil samples instead of one (every soil sample was compacted once). All the tests were carried out in triplicate and the results were adjusted using a polynomial line. Comparing the results from the second group of samples against the control values, the second technique caused a $1 \%$ decrease in the maximum dry specific gravities, and optimal humidity increase close to $5 \%$. These differences are caused by the process of preparing the soil samples in the Proctor test variant.

\section{Keywords}

Standard Proctor, dry specific weight, moisture content, re-compaction. 
$\mathrm{E}$ 1 objetivo básico de la compactación es mejorar las características del comportamiento de los suelos. Con esta técnica es posible disminuir la compresibilidad de los suelos e incrementar su estabilidad volumétrica ante cambios en su contenido de agua, además de obtener un aumento de la resistencia, rigidez y una disminución de la permeabilidad (Abeyrathne et al., 2019; Zhang et al., 2018; Hossain y Yin, 2010; Yin, 2009). El estado de compactación de una muestra de suelo está definido por dos variables de estado: el peso específico seco $\left(\gamma_{\mathrm{d}}\right)$ y el contenido de humedad (w). Cuando se añade agua al suelo durante la compactación, ésta actúa como agente lubricante sobre las partículas, deslizándose una sobre la otra, quedando densamente empaquetadas. Cuando el contenido de humedad se aumenta gradualmente y se mantiene el mismo esfuerzo compactador, el peso específico seco del suelo aumenta progresivamente hasta un máximo conocido como peso específico seco máximo $\left(\gamma_{\text {dmáx }}\right)$. Más allá de este límite, cualquier aumento en el contenido de agua tiende a reducir el peso específico seco (Figura 1). El contenido de humedad en el que se alcanza el peso específico seco máximo se denomina contenido de humedad óptimo ( $\mathrm{w}_{\mathrm{opt}}$ ) (Das: 2015).

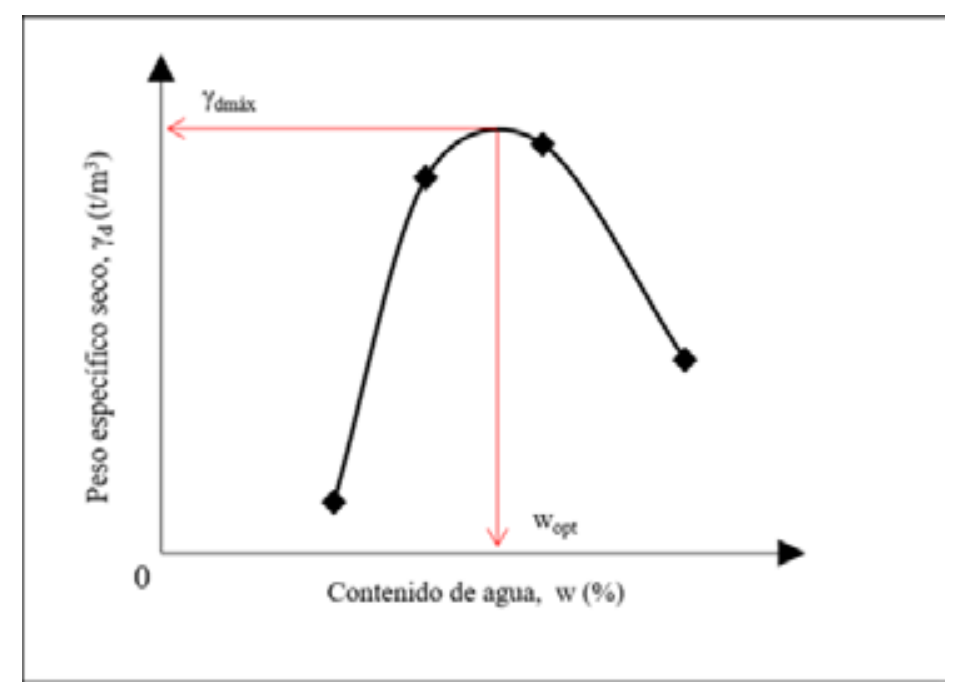

Figura 1. Curva de compactación Proctor estándar. Fuente: elaboración propia

Los métodos clásicos para determinar los parámetros de compactación, son los definidos por Ralph R. Proctor (1933): Ensayos Proctor Estándar y Proctor modificado (también conocidos como AASHTO estándar y AASHTO modificado, respectivamente). En lo que se refiere al primero de estos ensayos, el manual de Métodos de Muestreo y Pruebas de Materiales del Instituto Mexicano del Transporte, M-MMP-1-09 (2006), en su sección 09 (compactación AASHTO), describe el procedimiento para determinar 
mediante una curva de compactación el peso específico seco máximo y la humedad óptima. Los libros publicados por Juárez-Badillo y RicoRodríguez (2005), así como por Braja M. Das (2015) también describen el mencionado procedimiento.

De manera general, el procedimiento para realizar el ensayo Proctor Estándar se puede resumir de la siguiente manera: (i) separar por cuarteos una porción representativa de aproximadamente $4 \mathrm{~kg}$ de suelo; (ii) a la porción de suelo seleccionado se le agrega una cantidad de agua necesaria para que una vez homogenizado tenga un contenido de agua inferior en 4 a 6\% al óptimo estimado; (iii) esta porción de suelo se compacta dentro del molde de la prueba (Figura 2) en tres capas, aplicando a cada una 25 golpes con un pisón de $2.5 \mathrm{~kg}$ a una altura de caída de $30.48 \mathrm{~cm}$; y (iv) terminada la compactación de las capas se determina el peso específico del material compactado y su contenido de humedad. Con este procedimiento se consigue un punto de la curva de compactación. Es recomendable contar con al menos cuatro puntos para tener una curva bien definida (Figura 1).
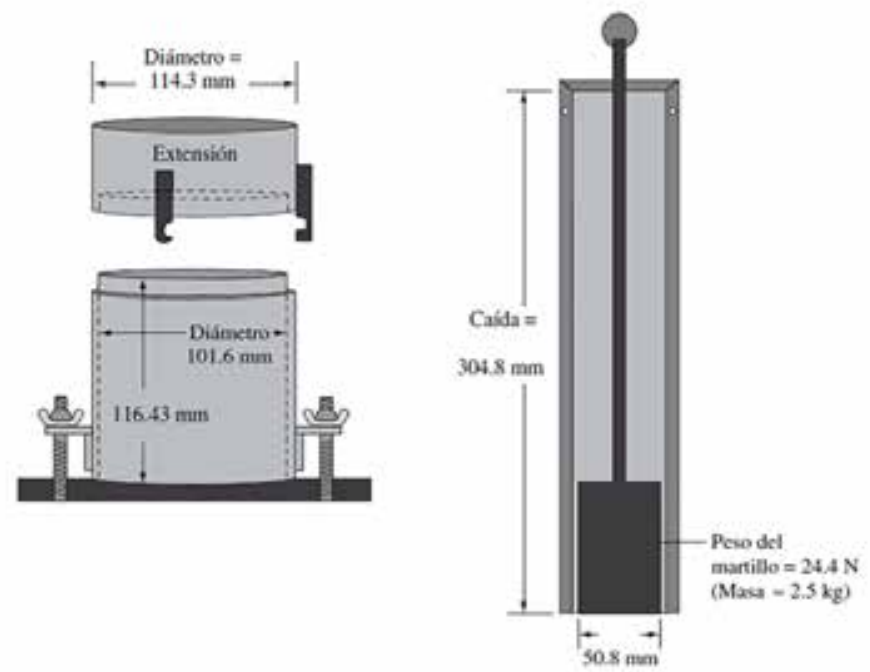

Figura 2. Equipo para el ensayo de compactación Proctor Estándar (Das: 2015). Fuente: elaboración propia

Para conseguir el siguiente punto, el manual M-MMP-1-09 (2006) señala que se utilice la misma porción de suelo con el que se realizó el primer punto y que se le incorpore aproximadamente $2 \%$ de agua con respecto a la masa inicial de la porción de prueba y se repitan los pasos (iii) y (iv) antes mencionados. Este procedimiento debe repetirse para cada punto. Es decir, la misma porción de suelo es utilizada varias veces. Las condiciones óptimas de compactación se determinan identificando el contenido de agua para el que se alcanza el peso específico seco máximo $\left(\gamma_{\mathrm{dmáx}} \mathrm{y} \mathrm{w}_{\mathrm{opt}}\right)$ (Figura 1). 
Existen diversos factores que influyen en el proceso de compactación, por ejemplo, Sivakumar y Wheeler (2000) estudiaron la influencia de la presión de compactación, el contenido de agua y del tipo de compactación en el comportamiento mecánico e hidráulico de una arcilla caolinita Blanca. Otros investigadores han estudiado el efecto del tipo de suelo (Mora-Ortiz et al., 2014; Izquierdo et al., 2011; Rico-Rodríguez y del Castillo, 1992), el contenido de agua ( Jiang et al., 2015; Duong et al., 2013), la temperatura ambiente durante el ensayo y el nivel de energía aplicada (Heitor et al., 2015; Mendoza: 1992). Sin embargo, en la bibliografía consultada no se mencionan los posibles efectos que tiene el utilizar una misma porción de suelo para todo el ensayo Proctor estándar.

En la práctica habitual de esta prueba en los laboratorios de materiales, es común utilizar varias porciones de suelo en lugar de una sola, es decir, para cada punto de la curva de compactación se utiliza una porción de suelo. Con lo anterior es posible reducir considerablemente los tiempos de ejecución del ensayo. Con la utilización de esta variante en el procedimiento del ensayo Proctor, surge una interrogante, ¿̇habrá algún cambio en el resultado del ensayo Proctor estándar si en lugar de usar una sola porción de suelo como indica el manual M-MMP-1-09 (2006) para determinar los parámetros de compactación se utilizan porciones independientes para cada punto de la curva de compactación? Esta investigación pretende aportar información acerca de la variación de los resultados obtenidos entre estas dos formas de realizar la prueba Proctor estándar.

\section{METODOLOGÍA}

Para llevar a cabo esta investigación se utilizaron dos diferentes tipos de suelos, los cuales se obtuvieron mediante el método PCA (pozo a cielo abierto) a una profundidad de $1.5 \mathrm{~m}$. A continuación, se muestra su información básica:

Suelo 1. Este suelo se obtuvo a un costado de la carretera Federal Villahermosa-Teapa en el entronque con la carretera estatal Playas del Rosario-Teapa, a la altura del km $020+285$ (Figura 3). El material extraído es de color rojo y no presenta contenido de materia orgánica. Las características básicas del suelo se muestran en la Tabla 1.

Suelo 2. Este suelo se extrajo a un costado de la carretera Federal Dos Bocas-Reforma, mejor conocido como vía corta en el municipio de Comalcalco, Tabasco, a la altura del Restaurante familiar Oasis (Figura 3). El material extraído es de un color café claro y no hay presencia de materia orgánica. Sus características básicas se muestran en la Tabla 1. 


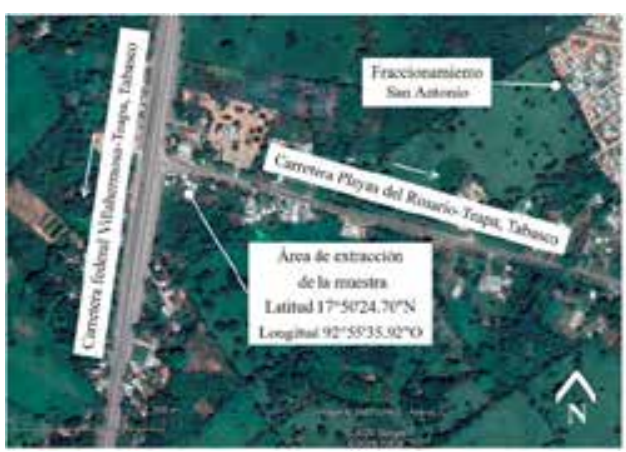

(a) Suelo 1

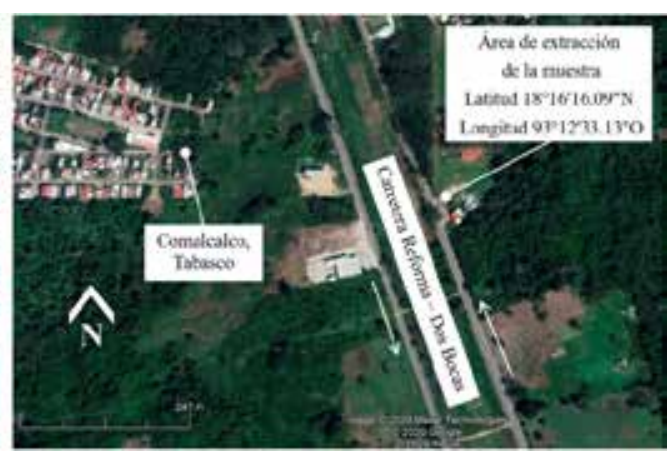

(b) Suelo 2

Figura 3. Zonas de extracción de los suelos en estudio. Fuente: Google maps

\section{Tabla 1}

Clasificación y características básicas de los suelos en estudio

\begin{tabular}{ccc}
\hline Propiedad & Suelo 1 & Suelo 2 \\
Límite liquido (LL) & $83 \%$ & $34.9 \%$ \\
Límite plástico (LP) & $17.33 \%$ & $25.62 \%$ \\
Índice de plasticidad (IP) & $65.66 \%$ & $9.28 \%$ \\
Peso específico relativo de sólidos (Ss) & $2.69 \%$ & 2.58 \\
Contenido de finos (\%) & 97.44 & 96.52 \\
Contenido de arena (\%) & 2.56 & 3.48 \\
Clasificación S.U.C.S* & CH (Arcilla de alta & ML (Limo de baja \\
& compresibilidad) & compresibilidad) \\
\hline
\end{tabular}

*Sistema Unificado de Clasificación de Suelos

Fuente: elaboración propia

Para determinar si existe una variación de los resultados entre los dos procedimientos de la prueba Proctor estándar, se siguió el procedimiento que a continuación se describe:

En primer lugar, se obtuvieron los parámetros óptimos de compactación $\left(\gamma_{\text {dmáx }} \mathrm{y} \mathrm{w}_{\text {opt }}\right)$ para ambos suelos siguiendo el procedimiento convencional que marca el Manual del Instituto Mexicano del Transporte (M-MMP-1-09: 2006), es decir, utilizando una sola porción de $4.5 \mathrm{~kg}$ de suelo para todo el ensayo (Figura 4). Estos resultados tuvieron la función de valores de referencia.

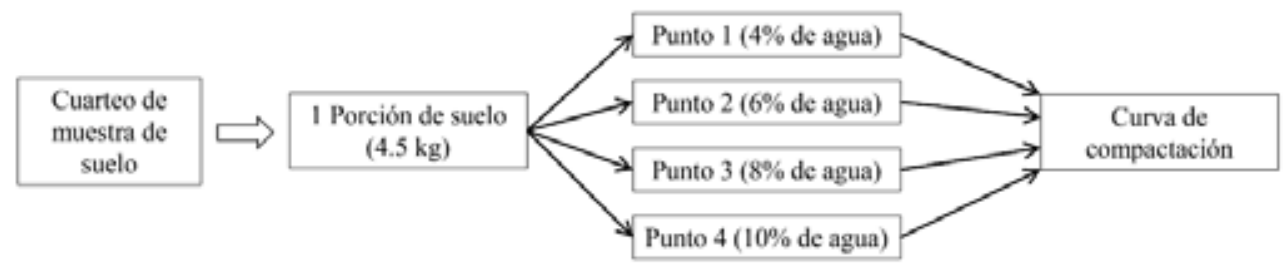

Figura 4. Ensayo referencia (una porción de suelo para todo el ensayo Proctor estándar). El \% de agua es con respecto a la masa inicial de la porción de prueba. Fuente: elaboración propia 
Todos los ensayos Proctor estándar en esta investigación se realizaron por triplicado y se ajustaron mediante una línea polinómica. Los ensayos se realizaron en un laboratorio con temperatura controlada $\left(24^{\circ} \mathrm{C}\right)$ vigilando que cada aspecto de su ejecución (incrementos de agua durante el ensayo, posición del pisón, distribución de los golpes, etc.) fuese el mismo para todos y acorde a los señalamientos del manual M-MMP-1-09 (2006). Los parámetros óptimos de compactación se determinaron de acuerdo con el método tradicional propuesto por Proctor (1933): identificando el contenido de agua para el que se alcanza el peso específico seco máximo.

1. Con los parámetros de compactación de referencia obtenidos, el siguiente paso fue repetir los ensayos Proctor estándar en ambos suelos, siguiendo el procedimiento que marca el Manual del Instituto Mexicano del Transporte (M-MMP-1-09: 2006), pero con la variante de utilizar una porción de $4.5 \mathrm{~kg}$ de suelo para cada punto de la curva de compactación (Figura 5), es decir, cada porción de suelo solo se compactó una vez para obtener un punto de la curva de compactación. Cada una de estas porciones se obtuvo mediante cuarteo. A cada porción se le agregó un porcentaje de agua, con respecto a la masa inicial de la porción de prueba, y se le dejó reposar en recipientes cerrados herméticamente durante 24 horas antes de compactarla.

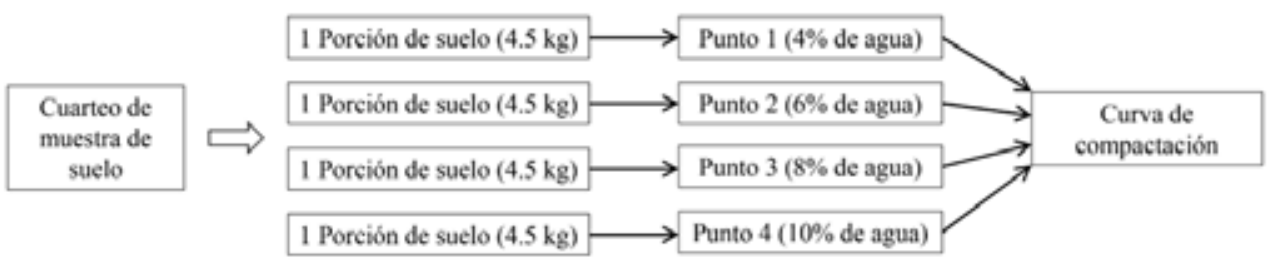

Figura 5. Ensayo con varias porciones de suelo (variante del procedimiento de compactación Proctor Estándar). El \% de agua es con respecto a la masa inicial de la porción de prueba. Fuente: elaboración propia

2. Por último, se compararon los resultados obtenidos con ambos procedimientos del ensayo Proctor estándar y se realizaron comentarios y conclusiones.

\section{RESULTADOS}

La Figura 6, muestra los resultados de los ensayos de compactación Proctor estándar, realizados sobre el suelo 1: a) ensayo convencional con una sola porción de suelo; b) ensayo Proctor que utiliza varias porciones de suelo; y c) comparación de las curvas de compactación obtenidas con ambos procedimientos. 
En la Figura 6(a) se observan las tres repeticiones del ensayo Proctor convencional realizado al suelo 1, cada uno de los tres ensayos se realizó de la misma manera. En cada ensayo se obtuvieron cinco puntos de la curva de compactación. Se observa que el punto uno en los tres ensayos tiene aproximadamente el mismo contenido de humedad $(w \approx 28.3 \%)$ y el mismo peso específico seco $\left(\gamma_{\mathrm{d}}=1.57 \mathrm{t} / \mathrm{m}^{3}\right)$. Sin embargo, a partir del punto dos hay ligeras variaciones entre los mismos puntos de cada ensayo, por ejemplo, si se comparan entre sí el punto tres de los tres ensayos se observa una diferencia máxima del 1\% en el contenido de humedad, por su parte, comparando el punto cinco en los tres ensayos la diferencia máxima del peso específico seco entre ellos es del $2 \%$. Se observa que la diferencia entre los mismos puntos de cada ensayo se acentúa conforme el contenido de agua se incrementa. Estas diferencias se presentaron a pesar de ejecutar la prueba Proctor estándar convencional con el mismo procedimiento, en las mismas condiciones y con los mismos incrementos de agua para cada punto (M-MMP-1-09: 2006). Lo anterior, es evidencia de que las variaciones en humedad y peso específico entre los mismos puntos de los tres ensayos están ligadas a la distribución del agua en la única porción de suelo que se usó para cada ensayo, y es que después de cada incremento de agua no se da ningún tiempo de reposo para que ésta se distribuya homogéneamente en la masa de suelo. Esta falta de tiempo repercute en la distribución uniforme del agua en la muestra, produciendo las variaciones que se presentan en la Figura.6(a).

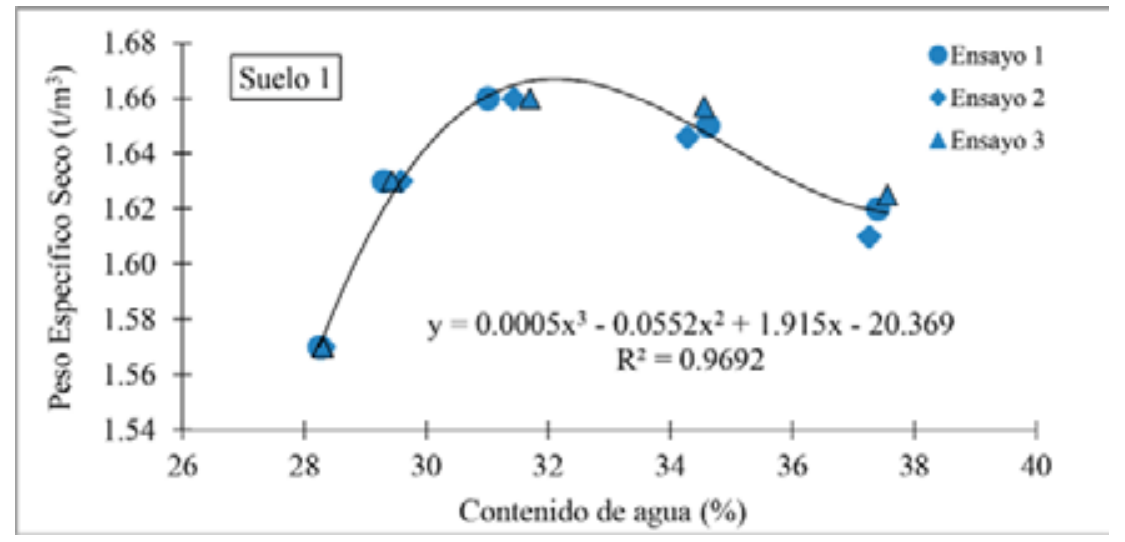

(a) Proctor estándar convencional (1 porción de suelo por ensayo) 


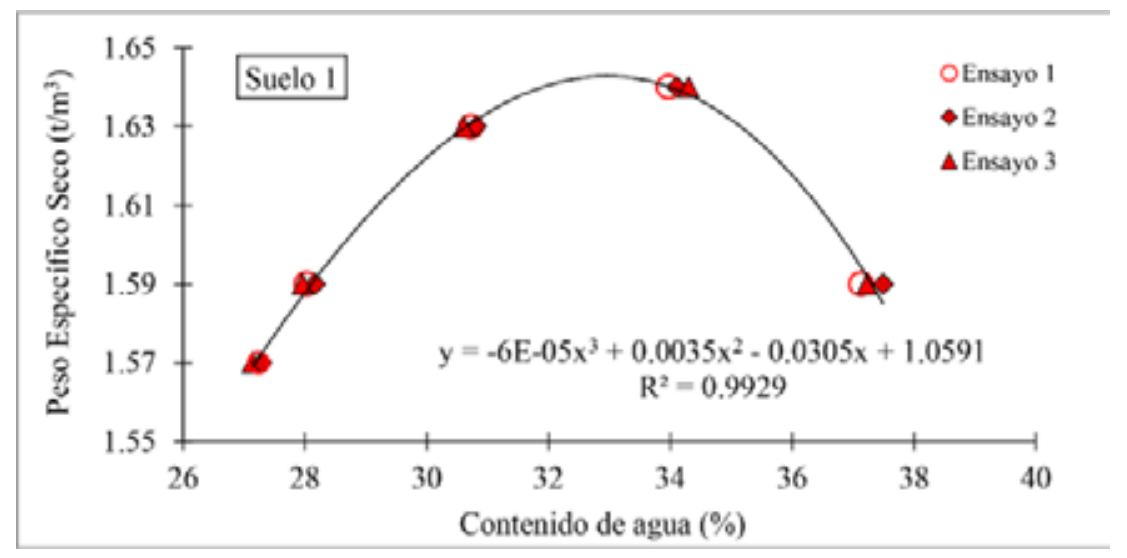

b) Proctor estándar con varias porciones de suelo por ensayo

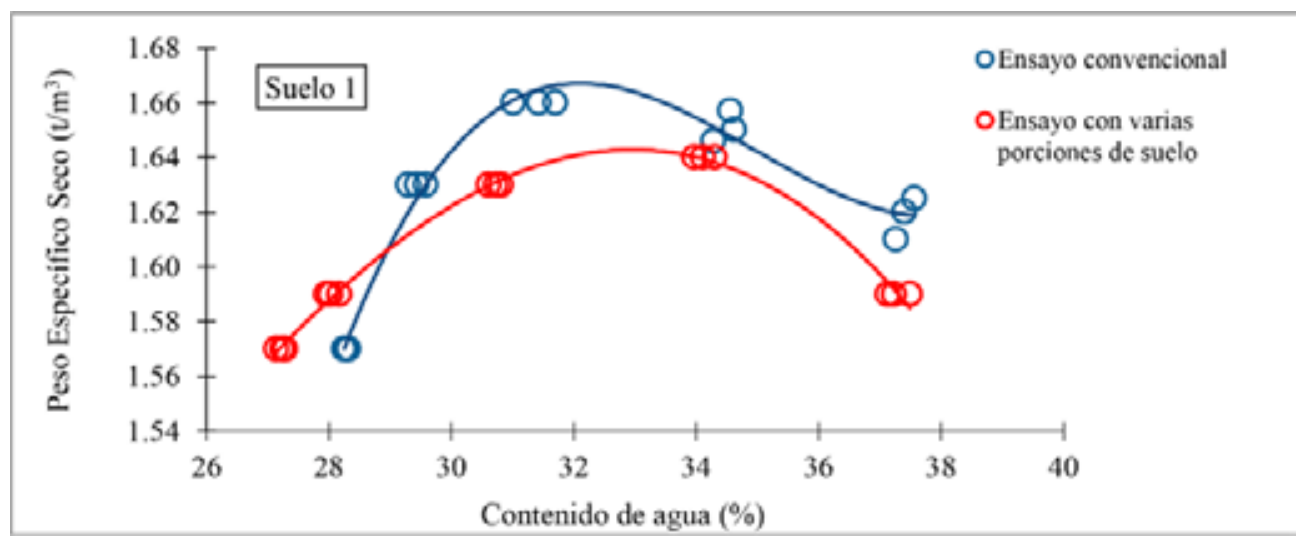

c) Curvas de compactación con ambas variantes del ensayo Proctor estándar

Figura 6. Pruebas Proctor Estándar para el suelo 1. Fuente: elaboración propia

Por su parte, la Figura 6(b) muestra las tres repeticiones del ensayo Proctor sobre el suelo 1 pero utilizando una porción de suelo para cada punto de la curva de compactación. Es posible observar una mayor correspondencia entre los valores de $w$ y $\gamma_{\mathrm{d}}$ para los mismos puntos de las tres repeticiones. Se observa que todos los pesos específicos secos son iguales entre sí para los mismos puntos de los tres ensayos. Sin embargo, en el contenido de humedad se registraron diferencias menores al $1 \%$ entre los mismos puntos de cada ensayo. Esta uniformidad en los valores del $\gamma_{\mathrm{d}}$ para los mismos puntos de los tres ensayos se debe al proceso de preparación de cada porción de suelo. Como se explicó anteriormente, en esta variante del ensayo Proctor estándar se utiliza una porción de suelo para cada punto de la curva de compactación. Cada porción recibe un contenido de humedad diferente y se deja reposar por 24 horas en recipientes sellados herméticamente (Figura 5) antes de 
compactar. El periodo de reposo para cada porción de suelo asegura que la humedad se homogenice en toda la muestra.

En la Figura 6(c) se comparan las curvas de compactación obtenidas con ambos procedimientos del ensayo Proctor estándar sobre el suelo 1, mientras que la Tabla 2 muestra los valores del peso específico seco máximo $\left(\gamma_{\text {dmáx }}\right)$ y la humedad óptima $\left(\mathrm{w}_{\mathrm{opt}}\right)$ de compactación determinadas con los ambos procedimientos.

Los resultados muestran que el procedimiento convencional generó pesos específicos secos mayores a humedades óptimas menores, con respecto a la variante que utiliza varias porciones de suelo. La diferencia entre los $\gamma_{\text {dmáx }}$ fue de $1.5 \%$, mientras que para las $\mathrm{w}_{\text {opt }}$ fue de $1 \%$.

En la Figura 7(a) se observan las tres repeticiones del ensayo Proctor convencional realizado al suelo 2, cada uno de los tres ensayos se realizó de la misma manera. En cada ensayo se obtuvieron cuatro puntos de la curva de compactación. Al igual que en el caso anterior, se observa que el punto uno en los tres ensayos tiene el mismo peso específico seco $\left(\gamma_{\mathrm{d}}=1.73 \mathrm{t} / \mathrm{m}^{3}\right)$ con ligeras variaciones en el contenido de humedad (1.31\% de diferencia máxima entre los puntos).

\section{Tabla 2}

Resultados de los ensayos Proctor Estándar para ambos suelos en estudio

\begin{tabular}{|c|c|c|c|c|}
\hline \multirow{2}{*}{$\begin{array}{l}\text { Parámetros de } \\
\text { compactación }\end{array}$} & \multicolumn{2}{|c|}{$\begin{array}{l}\text { Sueldo } 1 \\
\text { Arcilla de alta compresibilidad }\end{array}$} & \multicolumn{2}{|c|}{$\begin{array}{c}\text { Suelo } 2 \\
\text { Limo de baja compresibilidad }\end{array}$} \\
\hline & $\begin{array}{c}\text { Ensayo } \\
\text { convencional }\end{array}$ & $\begin{array}{l}\text { Ensayo con varias } \\
\text { porciones de suelo }\end{array}$ & $\begin{array}{c}\text { Ensayo } \\
\text { convencional }\end{array}$ & $\begin{array}{l}\text { Ensayo con varias } \\
\text { porciones de suelo }\end{array}$ \\
\hline $\begin{array}{l}\text { Peso específico seco } \\
\text { máximo }\left(\mathrm{t} / \mathrm{m}^{3)}\right.\end{array}$ & 1.668 & 1.643 & 1.781 & 1.772 \\
\hline Humedad óptima (\%) & 32 & 33 & 23.4 & 24.7 \\
\hline
\end{tabular}

Fuente: Elaboración propia

Al igual que en el suelo 1, se advierte que, comparando los mismos puntos en los tres ensayos, a partir del punto dos existen variaciones en los valores del peso específico seco y en el contenido de humedad. Estás variaciones se acentúan con el incremento en el contenido de agua. Las máximas diferencias se presentan en los puntos dos y cuatro, con magnitudes máximas del $2 \%$ en el contenido de humedad y del $1 \%$ en el peso específico seco.

La Figura 7(b) muestra las tres repeticiones con el suelo 2 de la variante del ensayo Proctor estándar que utiliza varias porciones de suelo. Nuevamente se observa una mayor correspondencia entre los valores de $w$ y $\gamma_{\mathrm{d}}$ para los mismos puntos de las tres repeticiones. Se advierte que todos los pesos específicos secos son iguales entre sí para cada punto de los tres ensayos. El contenido de agua entre los mismos puntos de los tres ensayos varía en 
menos del 1\%. Así como en el caso del suelo 1, se observa que la variante del ensayo Proctor muestra resultados más homogéneos entre los mismos puntos de los tres ensayos. Lo anterior se debe al proceso de preparación de la muestra, que, como se explicó anteriormente, permite una homogenización de la humedad dentro de la porción de suelo.

En la Figura 7 (c) se comparan las curvas de compactación obtenidas con ambos procedimientos del ensayo Proctor estándar sobre el suelo 2. La Tabla 2 muestra los valores óptimos de compactación $\left(\gamma_{\text {dmáx }}, \mathrm{w}_{\text {opt }}\right)$ determinados con los ambos procedimientos.

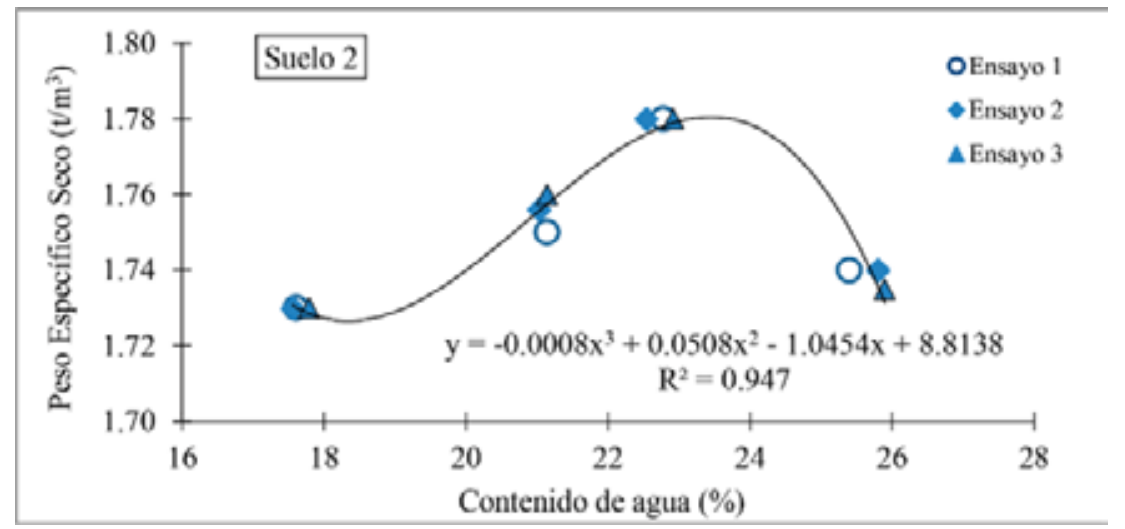

(a) Proctor estándar convencional (1 porción de suelo por ensayo)

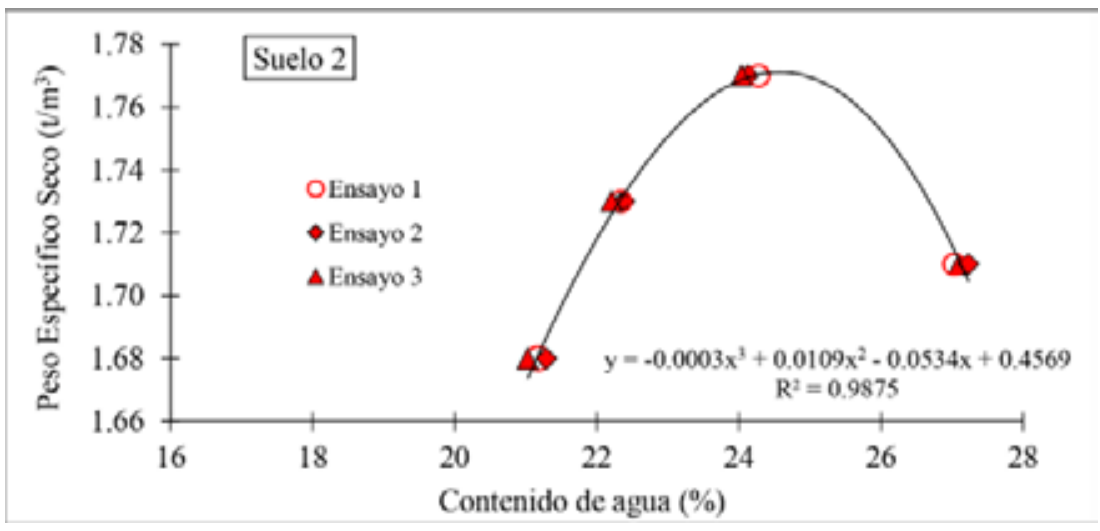

(b) Proctor estándar con varias porciones de suelo por ensayo 


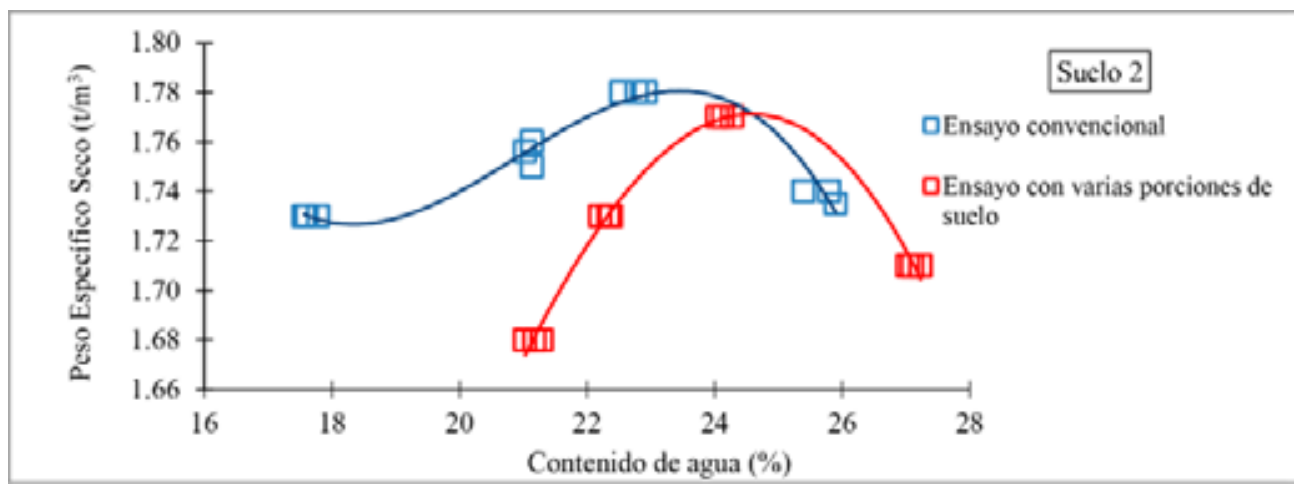

(c) Curvas de compactación con ambas variantes del ensayo Proctor estándar

Figura 7. Pruebas Proctor Estándar para el suelo 2. Fuente: elaboración propia

Comparando ambas curvas de compactación, se observa que el procedimiento convencional generó pesos específicos secos mayores a humedades óptimas menores, con respecto a la variante que utiliza varias porciones de suelo. La diferencia entre los $\gamma_{\text {dmáx }}$ fue de $0.51 \%$, mientras que para las $\mathrm{w}_{\text {opt }}$ fue de $1.3 \%$.

\section{CONCLUSIONES}

Los resultados experimentales demostraron que, al menos para los suelos en estudio, realizar el ensayo Proctor Estándar con la variante de utilizar una porción de suelo para cada punto de la curva de compactación, genera pesos específicos secos máximos ligeramente menores con contenidos de agua óptimos, levemente mayores a los que se obtendrían con el ensayo Proctor Estándar convencional. Es interesante notar que si bien las diferencias entre los parámetros óptimos de compactación obtenidos con ambos procedimientos (Tabla 2) son pequeñas, también muestran un comportamiento constante. Es decir, siempre los ensayos Proctor realizados con varias porciones de suelo generaron un $\gamma_{\text {dmáx }}$ menor al que se obtuvo con el procedimiento convencional.

Durante la ejecución de ambos procedimientos del ensayo Proctor se pudo constatar que la variante que utiliza varias porciones de suelo se realiza más rápido que con el procedimiento convencional, debido a que no es necesario realizar incrementos de agua sobre una misma porción de suelo ni tampoco es necesario mezclar hasta homogenizar, ya que se cuenta con las porciones de suelo humedecidas previamente y, con un reposo de 24 horas, se garantiza la homogeneidad de la humedad. El tiempo que se ahorra en la ejecución de la prueba está en función del operador, pero sin duda, el ahorro de tiempo es un factor muy importante en el ámbito profesional. En adición, la diferencia entre las magnitudes de los pesos específicos secos máximos 
obtenidos con ambos procedimientos podría perder importancia desde el punto de vista práctico, ya que esta diferencia es menor al $1.5 \%$ en magnitud.

Es necesario realizar estudios más extensos sobre este tema, una segunda fase de esta investigación será ensayar otros tipos de suelos con diferentes contenidos de arena, más repeticiones de los ensayos, diferentes energías de compactación y con otros métodos de compactación, como por ejemplo el Harvard miniatura. 


\section{REFERENCIAS}

Abeyrathne, A., Sivakumar, V., Kodikara, J. (2019). Isotropic volumetric behaviour of compacted unsaturated soils within specific volume, specific water volume, mean net stress ( v , v w , p ) space. Canadian Geotechnical Journal, 56(12), 1756-1778. https://doi.org/10.1139/ cgj-2018-0230

Das, B. M. (2015). Fundamentos de Ingeniería Geotécnica. Thomson Learning (ed.); Cuarta edición.

Duong, T. V., Tang, A. M., Cui, Y.-J., Trinh, V. N., Dupla, J.-C., Calon, N., Canou, J., Robinet, A. (2013). Effects of fines and water contents on the mechanical behavior of interlayer soil in ancient railway sub-structure. Soils and Foundations, 53(6), 868-878. https://doi. org/10.1016/j.sandf.2013.10.006

Heitor, A., Indraratna, B., Rujikiatkamjorn, C. (2015). The role of compaction energy on the small strain properties of a compacted silty sand subjected to drying-wetting cycles. Géotechnique, 65(9), 717727. https://doi.org/10.1680/geot.14.P.053

Hossain, M. A., Yin, J.-H. (2010). Shear strength and dilative characteristics of an unsaturated compacted completely decomposed granite soil. Canadian Geotechnical Journal, 47(10), 1112-1126. https://doi.org/10.1139/T10-015

Izquierdo, M., Querol, X., Vazquez, E. (2011). Procedural uncertainties of Proctor compaction tests applied on Mswi bottom ash. Journal of Hazardous Materials, 186, 1639-1644. https://doi.org/10.1016/j. jhazmat.2010.12.045

M-MMP-1-09/06. мMP. (2006). Métodos de muestreo y prueba de materiales. Suelos y materiales para terracerías: Compactación AASHTO. Secretaría de Comunicaciones y Transportes. Instituto Mexicano del Transporte, IMT. https://bit.ly/30JRcoq

Jiang, H., Bian, X., Chen, Y., Han, J. (2015). Impact of Water Level Rise on the Behaviors of Railway Track Structure and Substructure. Transportation Research Record: Journal of the Transportation Research Board, 2476(1), 15-22. https://doi.org/10.3141/2476-03

Juárez-Badillo, E., Rico-Rodríguez, A. (2005). Mecánica de Suelos. Fundamentos de la Mecánica de Suelos. Tomo 1 (E. L. S. A. de C. V. G. N. E. México. (ed.)).

Mendoza lópez, M. (1992). Enfoques recientes en la compactación de suelos. Publicación Técnica No. 33. Secretaría de Comunicaciones y Transportes. Instituto Mexicano del Transporte. Querétaro, Qro. https://bit.ly/3fNSrHD 
Mora-Ortiz, R. S., Romero, E., Baptista, A. (2014). Evolución de la microestructura de un suelo limoso compactado a lo largo de trayectorias hidro-mecánicas.X. R. N. de M. de S. e I. Geotécnica ed. http://hdl.handle.net/2117/99958

Proctor, R. R. (1933). Design and Construction of Rolled Earth Dams. Engineering News Record, 3, 245-248, 286-289, 348-351, 372-376.

Rico Rodríguez, A., del Castillo Mejía, H. (1992). Consideraciones sobre Compactación de suelos en Obras de infraestructura de Transporte. Documento Técnico No. 7. Secretaria de Comunicaciones y Transportes. Instituto Mexicano del Transporte. Sanfandila, Qro. https://bit.ly/3fNiGoX

Sivakumar, V., Wheeler, S. J. (2000). Influence of compaction procedure on the mechanical behaviour of an unsaturated compacted clay. Part 1: Wetting and isotropic compression. Géotechnique, 50(4), 359-368. https://doi.org/10.1680/geot.2000.50.4.359

Yin, J.-H. (2009). Influence of relative compaction on the hydraulic conductivity of completely decomposed granite in Hong Kong. Canadian Geotechnical Journal, 46(10), 1229-1235. https://doi.org/10.1139/To9-053

Zhang, T. W., Cui, Y. J., Lamas-Lopez, F., Calon, N., Costa D’Aguiar, S. (2018). Compacted soil behaviour through changes of density, suction, and stiffness of soils with remoulding water content. Canadian Geotechnical Journal, 55(2), 182-190. https://doi.org/10.1139/cgj-2016-0628 